\title{
Evaluation of PTEN Inhibitor Following Spinal Cord Injury on Recovery of Voiding Efficiency and Motor Function Observed by Regeneration in Spinal Cord
}

\author{
Young Sam Cho ${ }^{1}$, Su Jin $\mathrm{Kim}^{2}$, Khae Hawn Kim³ \\ ${ }^{1}$ Department of Urology, Kangbuk Samsung Hospital, Sungkyunkwan University School of Medicine, Seoul, Korea \\ ${ }^{2}$ Department of Urology, Yonsei University Wonju College of Medicine, Wonju, Korea \\ ${ }^{3}$ Department of Urology, Chungnam National University Sejong Hospital, Chungnam National University College of Medicine, Sejong, Korea
}

Purpose: Neurogenic bladder (NB) associated with spinal cord injury (SCI) is a serious health problem. However, no effective treatment has been developed for SCI patients with NB. Phosphatase and tensin homolog deleted on chromosome 10 (PTEN) inhibitors have been proposed as a promising option for inducing neural regeneration. Therefore, we investigated the effects of a tissue gene nerve (TGN), PTEN inhibitor, on voiding function, motor function, and the expression of growth factors after SCI.

Methods: In this experiment, female rats were randomly divided into 3 groups ( $\mathrm{n}=10$ in each group): the sham-operation group, the SCI-induced group, and the SCI-induced and TGN-treated group. Cystometry; the Basso, Beattie, and Bresnahan (BBB) scale test; the ladder walking test; hematoxylin and eosin staining; and Western blotting for brain-derived neurotrophic factor (BDNF), vascular endothelial growth factor (VEGF), and nerve growth factor (NGF) were performed to evaluate functional and molecular changes.

Results: After SCI, the rats exhibited decreased walking ability according to the BBB scale test and impaired coordinative function according to the ladder walking test. The PTEN inhibitor promoted enhanced walking ability and coordinative function. Cystometry showed voiding impairment after SCI and improved voiding function was observed after PTEN treatment. Overexpression of VEGF, BDNF, and NGF were observed after SCI. Administration of PTEN inhibitors significantly attenuated the overexpression of growth factors due to SCI.

Conclusion: PTEN inhibitor treatment diminished the overexpression of growth factors and promoted the repair of damaged tissue. PTEN inhibitor-treated rats also showed improved motor function and improved voiding function. Therefore, we suggest TGN as a new therapeutic agent that can be applied after SCI.

Keywords: Spinal cord injury; Phosphatase and tensin homolog deleted on chromosome 10; Cystometry; Motor function; Growth factor

\footnotetext{
- Fund/Grant Support: This work was supported by the National Research Foundation of Korea (NRF) grant funded by the Korea government (MSIT) (NRF-2017R1A2B2005412). This work was supported by research grants funded by the KOLON TissueGene, Inc.

- Research Ethics: The experimental procedures were performed in accordance with the animal care guidelines of the National Institutes of Health, and were approved by the Institutional Animal Care and Use Committee (IACUC) of Kyung Hee University [KHUASP[SE]-17-093].

- Conflict of Interest: YSC, SJK, and KHK, associate editors of International Neurourology Journal, are the authors of this article. However, they played no role whatsoever in the editorial evaluation of this article or the decision to publish it. Except for that, no potential conflict of interest relevant to this article was reported.
}

Corresponding author: Khae Hawn Kim (iD https://orcid.org/0000-0002-7045-8004 Department of Urology, Chungnam National University Sejong Hospital, Chungnam National University College of Medicine, 20 Bodeum 7-ro, Sejong 30099, Korea

E-mail:kimcho99@cnuh.co.kr

Submitted: September 30, 2020 / Accepted after revision: November 7, 2020
This is an Open Access article distributed under the terms of the Creative Commons Attribution Non-Commercial License (http://creativecommons.org/licenses/by-nc/4.0/) which permits unrestricted non-commercial use, distribution, and reproduction in any medium, provided the original work is properly cited. 


\section{- HIGHLIGHT}

- Neuroregeneration induced by PTEN inhibitor (TGN) improves voiding function in rat with spinal cord injury.

\section{INTRODUCTION}

Spinal cord injury (SCI) is a serious form of trauma that may cause severe or permanent disability. SCI induces primary mechanical damage followed by secondary damage to the spinal cord. The primary damage of SCI results from direct mechanical tissue disruption immediately after trauma, while the secondary damage is mediated by complex cellular and molecular processes. There is no gold standard treatment for patients with SCI. Even though various treatment methods with various cell types have been applied to SCI patients, no method has not yet to be identified as effective [1-3].

Neurogenic bladder (NB) is common health problem associated with SCI. Most SCI patients experience voiding dysfunction and failure of normal urination. Moreover, SCI patients are at a high risk for NB-associated adverse events such as urinary tract infection and urinary stones. Numerous attempts have been made to treat NB; however, effective treatment for $\mathrm{NB}$ does not exist at the present [4-6]. NB in SCI patients is induced by neuronal damage, and many preclinical and clinical studies have explored the possibility of using stem cells and other biomaterials to stimulate the regeneration of injured neural tissue [7-9]. However, the efficacy of stem cell therapy is insufficient and a novel approach is necessary.

In recent years, phosphatase and tensin homolog deleted on chromosome 10 (PTEN) inhibitors have emerged as a promising therapy for neural regeneration. PTEN has attracted keen attention for its regulation of axonal regrowth in the central and peripheral nervous systems. PTEN inhibitors have been used to facilitate neuroprotection and axonal outgrowth following lesions to dorsal root ganglion neurons, retinal ganglion cells, cortical neurons, and the corticospinal tract of the spinal cord $[10,11]$. Therefore, in the present study, we investigated the effects of a tissue gene nerve (TGN), PTEN inhibitor, on voiding function, motor function, and the expression of growth factors in the spinal cord.

\section{MATERIALS AND METHODS}

\section{Animals and Grouping}

Adult male Sprague-Dawley rats, weighing $250 \pm 10 \mathrm{~g}$ (12 weeks old, $n=30$ ), were obtained from a commercial breeder (Orient
Co., Seoul, Korea). Rats were randomly divided into the following 3 groups ( $\mathrm{n}=10$ each group): Sham-operation group, SCIinduced group, SCI-induced and TGN-treated group. The experimental procedures were performed in accordance with the animal care guidelines of the National Institutes of Health, and were approved by the Institutional Animal Care and Use Committee (IACUC) of Kyung Hee University [KHUASP[SE]-17-093].

\section{Inducing $\mathrm{SCl}$ and Treatment}

SCI model was induced according to the previously described method [12]. The rats were anesthetized by inhalation of isoflurane (2\% isoflurane in $30 \% \mathrm{O}_{2}$ and $70 \% \mathrm{~N}_{2}$, JW Pharmaceutical, Seoul, Korea) during surgery. A laminectomy was performed to expose the spinal cord at thoracic level T9-10 without disrupting the dura. A contusion injury was created using the New York University Impactor System (NYU impactor, New York, NY, USA) by dropping a 10-g impactor from $2.5-\mathrm{cm}$ height onto the exposed dura. To prevent hypothermia during surgery, body and rectal temperature was maintained at $36^{\circ} \mathrm{C} \pm 0.5^{\circ} \mathrm{C}$ during surgery using a Homeothermic Blanket Control Unit (Harvard Apparatus, Holliston, MA, USA) that enveloped the body and the head. In addition, it was monitored for an additional 2 hours after surgery. The animals in the sham-operation group were treated identically, except that the spinal cords were not damaged after the skin incisions.

Starting 3 days after the induction of SCI, the TGN-treated group was administered TGN (Kolon TissueGene, Inc. Rockville, MD, USA) once every 2 days and 7 times directly to the SCI site for 14 days (Fig. 1).

\section{BBB Scale Test}

Functional analysis was first assessed using the Basso, Beattie, and Bresnahan (BBB) locomotor scale according to previously established behavior tests [13]. The analysis was performed at 7, 11 , and 15 days after SCI induction. Four researchers blinded to the experimental groupings observed each subject's ambulation, gait, limb movement coordination, paw position and space, tail activity and body stability in a noise-free, open field arena for $5 \mathrm{~min}$.

\section{Horizontal Ladder Walking Test}

To evaluate changes in motor function and coordination, a hor- 


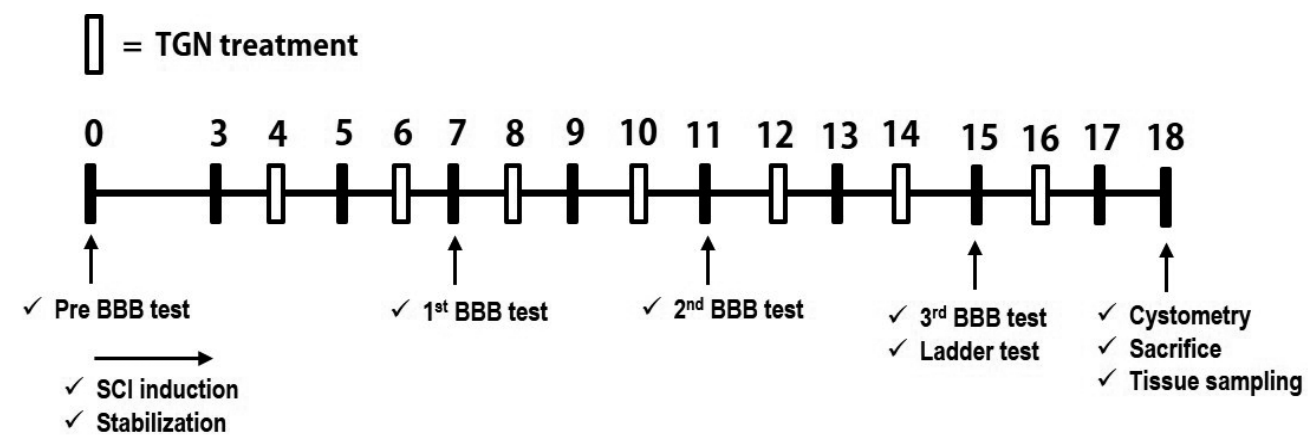

Fig. 1. Treatment schedule with PTEN inhibitor. PTEN, phosphatase and tensin homolog deleted on chromosome 10; BBB, Basso, Beattie, and Bresnahan; SCI, spinal cord injury.

izontal ladder walking was conducted according to previously study method [14]. The test was measured on the 15th day of the induction of SCI (after the 6th TGN treatment). Briefly, each experimental animal was allowed to cross a $1.5-\mathrm{m}$-long ladder rod designed with a $2-\mathrm{cm}$ spacing between round metal rods. While walking the ladder, it was evaluated whether the animal's hind legs were positioned correctly, and whether the fore and hind paws were organically coordinated. When the number of points cannot be moved, the maximum number of mistakes is 20. Depending on the number of mistakes, 0 to 1 is 10 points, 2 to 5 is 7 points, 6 to 9 is 4 points, and 10 to 20 is 1 point was given.

\section{Cystometry}

The voiding function was evaluated by cystometry 18 days after the surgery, as previously described [15]. The rats were anesthetized with Zoletil 50 (10 mg/kg, intraperitoneally; Vibac Laboratories, Carros, France). A sterile polyethylene catheter (PE50) with a cuff was implanted in the bladder through an abdominal midline incision into the dome and held in place by a pursestring suture. The catheter was connected to a pressure transducer (Harvard Apparatus) and syringe pump (Harvard Apparatus) via a 3-way stopcock to record the intravesical pressure and to infuse saline into the bladder. After the bladder was emptied, cystometry was performed by infusing $0.5 \mathrm{~mL}$ of saline. The bladder and voiding functions were monitored using Labscribe software (iWorx/CB Science Inc., Dover, DE, USA).

\section{Tissue Preparation}

Immediately after the cystometry, experimental animals were sacrificed for tissue collection. Tissue preparations were performed as previously described $[15,16]$. The rats were anesthetized using Zoletil 50 (10 mg/kg, interperitoneally; Virbac Lab- oratories). The rats were transcardially perfused with $50 \mathrm{mM}$ phosphate-buffered saline, followed by $4 \%$ paraformaldehyde in $100 \mathrm{mM}$ sodium phosphate buffer at $\mathrm{pH}$ 7.4. The spinal cord was removed, postfixed in the same fixative overnight, and transferred into a $30 \%$ sucrose solution for cryoprotection. Serial $40-\mu \mathrm{m}$-thick horizontal sections were made with a freezing microtome (Leica, Wetzlar, Germany). The spinal cord was selected from the region spanning damage site. Four sections on average in each region were collected from each rat.

\section{Analysis of Histological Changes With H\&E Staining} Hematoxylin and eosin (H\&E) staining was conducted as previous described [17]. The slides were immersed in Mayer's hematoxylin (DAKO, Glostrup, Denmark) for 1 minute, rinsed with tap water until clear, dipped in eosin (Sigma Chemical Co., St. Louis, MO, USA) for 20 seconds, and again rinsed with water. The slides were dipped twice in the following solutions: $95 \%$ ethanol, $100 \%$ ethanol, $50 \%$ ethanol, 50\% xylene solution, and $100 \%$ xylene. Finally, coverslips were mounted using Permount (Fisher Scientific, Waltham, MA, USA).

Images of $\mathrm{H} \& \mathrm{E}$ stained slides were taken with an Image-Pro plus computer-assisted image analysis system (Media Cyberbetics Inc., Silver Spring, MD, USA) attached to a light microscope (Olympus BX61, Olympus Corp., Tokyo, Japan). Inspectors who did not know the identity of the slide evaluated the image.

\section{Western Blotting}

Western blotting was performed according to the previously described method [18]. The bladder tissues were homogenized on chilled RIPA buffer (Cell Signaling Technology, Inc., Danvers, USA) with 1mM PMSF (Sigma Aldrich, ST Louis, MO, USA) and then centrifuged at $14,000 \mathrm{rpm}$ for 30 minutes at $4^{\circ} \mathrm{C}$. 
Protein contents were measured using a $\mu$-drop reader (Thermo Fisher Scientific, Vantaa, Finland). Next, 30- $\mu$ g protein was separated on sodium dodecyl sulfate-polyacrylamide gel electrophoresis gels and transferred onto a nitrocellulose membrane. The primary antibodies included the following: anti-mouse NGF antibody, anti-mouse VEGF antibody, anti-rabbit BDNF antibody (1:1,000; Santa Cruz Biotechnology, CA, USA).

The secondary antibodies were as follows: horseradish peroxidase (HRP)-conjugated anti-mouse antibody (1:5,000; Vector Laboratories, Burlingame, CA, USA) for NGF, VEGF; antirabbit antibody (1:5,000; Vector Laboratories) for BDNF. Blot membranes were detected using HRP-conjugated IgG (1:2,000; Vector Laboratories) and an enhanced chemiluminescence detection kit (Bio-Rad, Hercules, CA, USA). To compare the relative protein expressions, the detected bands were calculated densitometrically using Image-Pro plus computer-assisted image analysis system (Media Cybernetics Inc.). For relative quantification, the result in the sham-operation group was set as 1.00 .

\section{Data Analysis}

The data are expressed as the mean \pm standard error of the mean. For comparisons between groups, 1-way analysis of variance and the Duncan post hoc test were performed, and P-values $<0.05$ were considered to indicate statistically significant differences among the groups.

\section{RESULTS}

\section{Changes of Function Recovery (BBB Scale and Ladder Test)}

The functional recovery from BBB test are presented in Fig. 2A. Induction of SCI decreased BBB open field locomotor score in $\mathrm{BBB}$ test compared to sham-operation group $(\mathrm{P}<0.05)$. However, TGN treatment improved SCI-induced functional imbal-

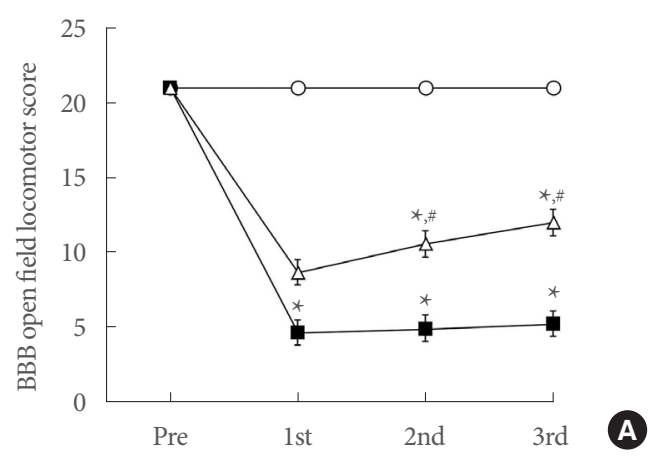

ance with increased BBB open field locomotor score. The improvement effect by TGN treatment increased as the number of injections.

Fig. 2B shows the motor function and coordination ability analysis results from the horizontal ladder test. Induction of SCI decreased ladder walking score, whereas TGN treatment enhanced the decreased ladder walking score by SCI. These results were given that TGN administration promote to recovery SCI by increasing motor function and coordination, which was reduced by SCI.

\section{Changes of Voiding Function in Cystometry}

The voiding function from cystometry are presented in Fig. 3. Induction of SCI increased bladder contraction pressure (CP), contraction time (CT), and intercontractional interval (ICI). After SCI injury, CP and CT were significantly decreased compared with the sham group $(\mathrm{P}<0.05)$. ICI of the SCI group was significantly increased compared with the sham group $(\mathrm{P}<$ 0.05). After TGN administration, CP and CT were significantly increased compared with the SCI group $(\mathrm{P}<0.05)$. ICI of the TGN-treared group was significantly decreased compared with the SCI group $(\mathrm{P}<0.05)$. Compared with the sham group, significant differences of CP, CT, and ICI were observed after TGN administration $(\mathrm{P}<0.05)$.

\section{Changes of Histology in Spinal Cord Tissue}

The appearance of histological change in spinal cord tissue at 18 days after induction of SCI shown in Fig. 4. The normal shape spinal cord tissue was observed in the sham-operation group. In the SCI group, histological picture showed the completely disrupted lesion in the dorsal area. However, TGN treatment decreased the SCI-induced disrupted lesion, and new tissues were increased around the damaged tissues.

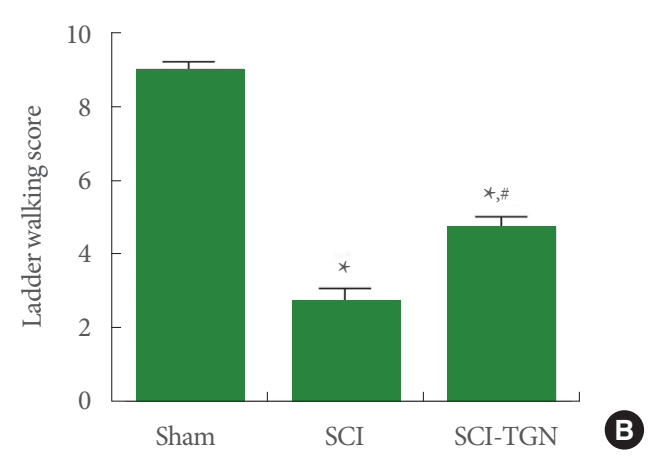

Fig. 2. Basso, Beattie, and Bresnahan (BBB) locomotor scale test (A) and horizontal ladder walking test (B). SCI, spinal cord injury; SCITGN, SCI with tissue gene nerve (TGN) treatment. ${ }^{*} \mathrm{P}<0.05$ compared with sham, ${ }^{*} \mathrm{P}<0.05$ compared with SCI. 

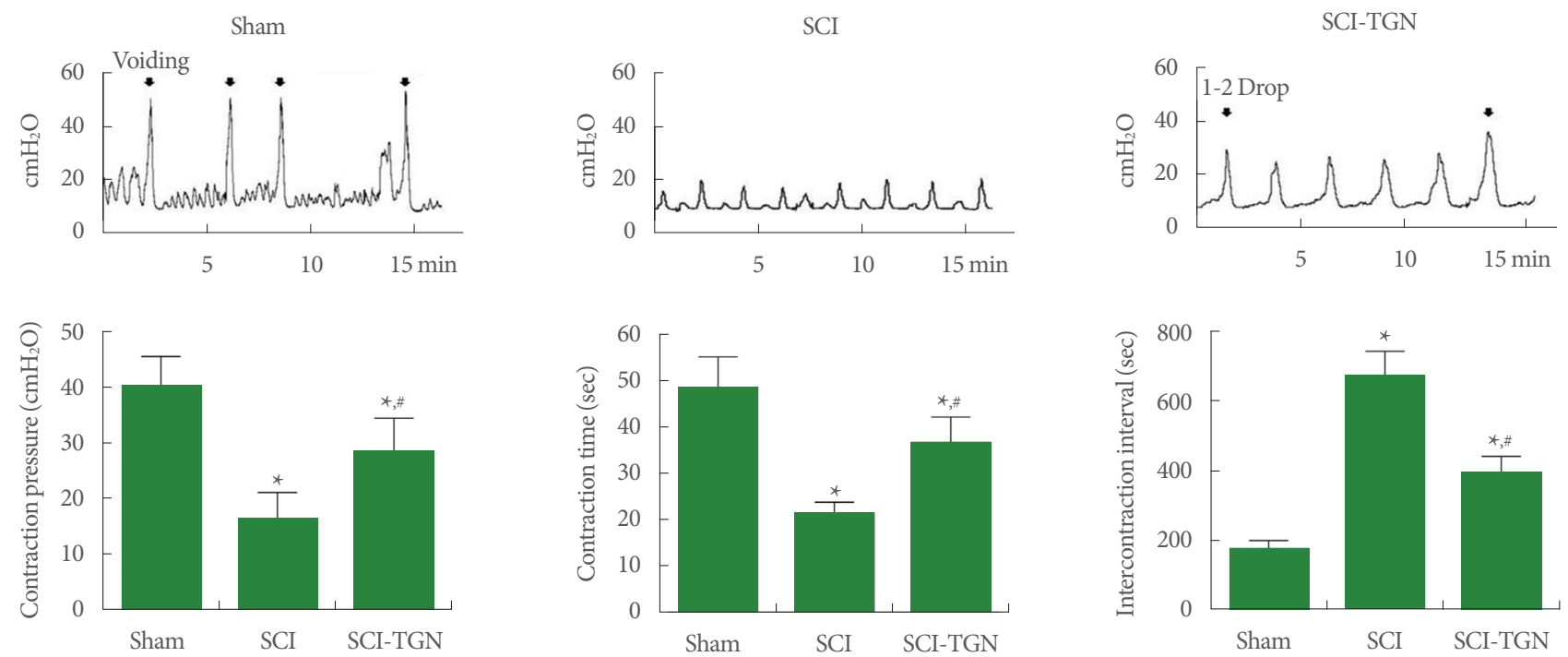

Fig. 3. Cystometric results. SCI, spinal cord injury; SCI-TGN, SCI with tissue gene nerve (TGN) treatment. ${ }^{\star} \mathrm{P}<0.05$ compared with sham, ${ }^{\#} \mathrm{P}<0.05$ compared with SCI.

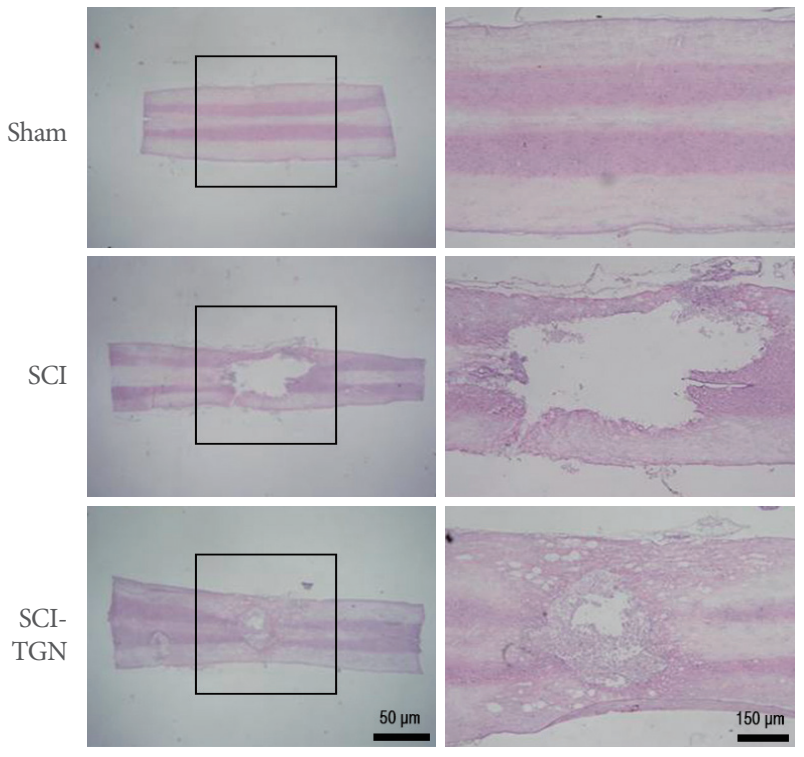

Fig. 4. Histologic finding. SCI, spinal cord injury; SCI-TGN, SCI with tissue gene nerve (TGN) treatment.

\section{Changes of VEGF, NGF, and BDNF Expressions in Bladder Tissue}

We performed western blotting to determine if TGN treatment improved SCI by examining its effect on VEGF, NGF, and BDNF expression (Fig. 5). Induction of SCI increased VEGF, NGF, and BDNF expression in spinal injury site tissue $(\mathrm{P}<$ 0.05). However, TGN treatment suppressed the expression of VEGF, NGF, and BDNF overexpressed in SCI induction $(\mathrm{P}<$
0.05). These results indicate that treatment of TGN suppresses the excessive compensatory response that is increased by SCI induction.

\section{DISCUSSION}

The present study demonstrated the effect of a PTEN inhibitor on functional and molecular impairment after SCI. TGN treatment improved walking ability and coordinative function after SCI. Moreover, the disappearance of normal voiding behavior induced by SCI was significantly restored after TGN treatment. However, the improvement of functional recovery did not reach the normal function observed in the sham group. Histologic recovery of the injured spinal cord was observed after TGN treatment. In addition, significantly lower NGF and BDNF levels were noted, and these findings suggest that the PTEN inhibitor stimulated neural recovery.

Several molecules are involved in the regeneration of neurons, of which TGN is considered to be one of the most directly implicated. Previous studies reported that tumor suppressor PTEN knockout mice showed significant regrowth of central nervous system axons after injury $[19,20]$. Thus, we chose PTEN as a new candidate for neural regeneration. The PI3K/ Akt pathway plays an important role in new axon formation and regeneration, and the overexpression of Akt contributes to neural regeneration and branching. PTEN reduces Akt activity; therefore, suppression of PTEN increases neural regeneration 



Fig. 5. Expression of VEGF (A), NGF (B), and BDNF (C). SCI, spinal cord injury; SCI-TGN, SCI with tissue gene nerve (TGN) treatment; VEGF, vascular endothelial growth factor; NGF, nerve growth factor; BDNF, brain-derived neurotrophic factor. ${ }^{\star} \mathrm{P}<0.05$ compared with sham, ${ }^{*} \mathrm{P}<0.05$ compared with $\mathrm{SCI}$.

by activating PI3K/Akt signaling [21]. Previous studies of PTEN inhibitors observed increased quantities of oligodendrocytes and recovery of motor function after cervical SCI [22]. Furthermore, infarction-associated functional impairment after cerebral artery occlusion infarction improved at a long-term follow-up after PTEN inhibitor treatment [23]. Similarly to these previous studies, the PTEN inhibitor used in this study could induce neural regeneration of the damaged spinal cord and functional improvement compared with the animals after SCI that did not receive PTEN inhibitor treatment. Moreover, voiding function improved in the present study. TGN treatment restored urination, with normal voiding patterns similar to those observed in the sham group.

Growth factors play an important role in tissue regeneration, and increased level of growth factors after any type of injury contribute to the recovery of damaged tissue. In this study, we compared levels of VEGF, NGF, and BDNF in each group. Significant overexpression of VEGF, NGF, and BDNF in the SCI group was considered to be a marker of the regeneration process. Wu et al. [24] and Sang et al. [25] showed that growth factors such as VEGF, NGF, and BDNF activated the PI3K/Akt pathway and induced neurogenesis.

However, functional studies on motor function and voiding showed functional impairment despite the overexpression of VEGF, NGF, and BDNF. Nonetheless, treatment with a PTEN inhibitor induced functional recovery and resulted in significantly lower expression of VEGF, NGF, and BDNF than found in the SCI animals. These results are associated with PTEN in- hibitor treatment because down-regulation of PTEN induced neural regeneration through the PI3K/Akt signaling pathway, without overexpression of growth factors.

This is the first study to investigate the role of a PTEN inhibitor in the recovery of voiding and motor function after SCI. However, it has some limitations. Based on the findings of this study, we suggest that the PI3K/Akt signaling pathway is the underlying mechanism. Although several previous studies have reported that PTEN inhibitors influenced the PI3K/Akt signaling pathway, changes in the PI3K/Akt signaling pathway were not specifically analyzed in this study.

In conclusion, PTEN inhibitors could be a new class of therapeutic candidates for functional impairment, including voiding dysfunction, in SCI patients. This is the first study to demonstrate both of the improvement of motor and voiding function after PTEN inhibitor treatment. However, our understanding of the precise mechanisms underlying the effects of PTEN inhibitors remains insufficient. Therefore, research on the pathophysiology of SCI and PTEN is necessary for PTEN inhibitors to be ready for clinical application.

\section{AUTHOR CONTRIBUTION STATEMENT}

- Conceptualization: KHK

- Data curation: SJK

- Formal analysis: YSC

- Funding acquisition: $K H K$

- Methodology: KHK 
- Project administration: KHK

- Visualization: SJK

-Writing-original draft: YSC

- Writing-review \& editing: YSC, KHK

\section{REFERENCES}

1. McDonald JW, Sadowsky C. Spinal-cord injury. Lancet 2002;359: 417-25.

2. Witiw CD, Fehlings MG. Acute spinal cord injury. J Spinal Disord Tech 2015;28:202-10.

3. Fakhoury M. Spinal cord injury: overview of experimental approaches used to restore locomotor activity. Rev Neurosci 2015;26:397-405.

4. Jeong SJ, Oh SJ. The current positioning of augmentation enterocystoplasty in the treatment for neurogenic bladder. Int Neurourol J 2020;24:200-10.

5. Nseyo U, Santiago-Lastra Y. Long-term complications of the neurogenic bladder. Urol Clin North Am 2017;44:355-66.

6. Bragge P, Guy S, Boulet M, Ghafoori E, Goodwin D, Wright B. A systematic review of the content and quality of clinical practice guidelines for management of the neurogenic bladder following spinal cord injury. Spinal Cord 2019;57:540-9.

7. Kim SJ, Cho YS, Park JM, Na YG, Kim KH. Stem cell therapy for neurogenic bladder after spinal cord injury: clinically possible? Int Neurourol J 2020;24(Suppl 1):S3-10.

8. Cho YS, Ko IG, Kim SE, Lee SM, Shin MS, Kim CJ, et al. Oral mucosa stem cells alleviates spinal cord injury-induced neurogenic bladder symptoms in rats. J Biomed Sci 2014;21:43.

9. Salehi-Pourmehr H, Hajebrahimi S, Rahbarghazi R, Pashazadeh F, Mahmoudi J, Maasoumi N, et al. Stem cell therapy for neurogenic bladder dysfunction in rodent models: a systematic review. Int Neurourol J 2020;24:241-57.

10. Christie KJ, Webber CA, Martinez JA, Singh B, Zochodne DW. PTEN inhibition to facilitate intrinsic regenerative outgrowth of adult peripheral axons. J Neurosci 2010;30:9306-15.

11. Zhao J, Qu Y, Wu J, Cao M, Ferriero DM, Zhang L, et al. PTEN inhibition prevents rat cortical neuron injury after hypoxia-ischemia. Neuroscience 2013;238:242-51.

12. Kim YM, Ji ES, Ko IG, Jin JJ, Cho YH, Seo TB. Combination of treadmill exercise with bone marrow stromal cells transplantation activates protein synthesis-related molecules in soleus muscle of the spinal cord injured rats. J Exerc Rehabil 2019;15:377-82.

13. Basso DM, Beattie MS, Bresnahan JC. A sensitive and reliable locomotor rating scale for open field testing in rats. J Neurotrauma
1995;12:1-21.

14. Schira J, Gasis M, Estrada V, Hendricks M, Schmitz C, Trapp T, et al. Significant clinical, neuropathological and behavioural recovery from acute spinal cord trauma by transplantation of a well-defined somatic stem cell from human umbilical cord blood. Brain 2012; 135:431-46.

15. Ko IG, Hwang L, Jin JJ, Kim SH, Han JH, Jeon JW, et al. Add-on therapy with the a-blockers tamsulosin and naftopidil improves voiding function by enhancing neuronal activity in prostatic hyperplasia rats. Int Neurourol J 2018;22:20-9.

16. Kim YM, Jin JJ, Lee SJ, Seo TB, Ji ES. Treadmill exercise with bone marrow stromal cells transplantation facilitates neuroprotective effect through BDNF-ERK1/2 pathway in spinal cord injury rats. J Exerc Rehabil 2018;14:335-40.

17. Lim H, Park SH, Kim SW, Cho KO. Therapeutic potential of human turbinate-derived mesenchymal stem cells in experimental acute ischemic stroke. Int Neurourol J 2018; 22(Suppl 3):S131-8.

18. Lee SS, Kim CJ, Shin MS, Lim BV. Treadmill exercise ameliorates memory impairment through ERK-Akt-CREB-BDNF signaling pathway in cerebral ischemia gerbils. J Exerc Rehabil 2020;16:49-57.

19. Park KK, Liu K, Hu Y, Smith PD, Wang C, Cai B, et al. Promoting axon regeneration in the adult CNS by modulation of the PTEN/ mTOR pathway. Science 2008;322:963-6.

20. Liu K, Lu Y, Lee JK, Samara R, Willenberg R, Sears-Kraxberger I, et al. PTEN deletion enhances the regenerative ability of adult corticospinal neurons. Nat Neurosci 2010;13:1075-81.

21. Ohtake Y, Hayat U, Li S. PTEN inhibition and axon regeneration and neural repair. Neural Regen Res 2015;10:1363-8.

22. Walker CL, Walker MJ, Liu NK, Risberg EC, Gao X, Chen J, et al. Systemic bisperoxovanadium activates Akt/mTOR, reduces autophagy, and enhances recovery following cervical spinal cord injury. PLoS One 2012;7:e30012.

23. Mao L, Jia J, Zhou X, Xiao Y, Wang Y, Mao X, et al. Delayed administration of a PTEN inhibitor BPV improves functional recovery after experimental stroke. Neuroscience 2013;231:272-81.

24. Wu H, Lu D, Jiang H, Xiong Y, Qu C, Li B, et al. Simvastatin-mediated upregulation of VEGF and BDNF, activation of the PI3K/Akt pathway, and increase of neurogenesis are associated with therapeutic improvement after traumatic brain injury. J Neurotrauma 2008;25:130-9.

25. Sang Q, Sun D, Chen Z, Zhao W. NGF and PI3K/Akt signaling participate in the ventral motor neuronal protection of curcumin in sciatic nerve injury rat models. Biomed Pharmacother 2018;103: $1146-53$. 